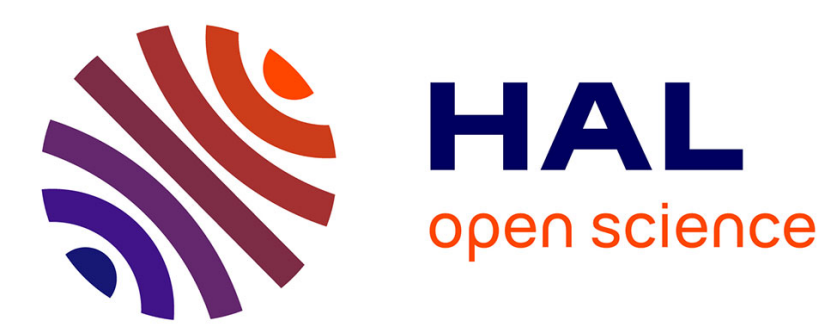

\title{
Distributed energy-efficient power optimization in cellular relay networks with minimum rate constraints
}

Giacomo Bacci, Elena Veronica Belmega, Luca Sanguinetti

\section{To cite this version:}

Giacomo Bacci, Elena Veronica Belmega, Luca Sanguinetti. Distributed energy-efficient power optimization in cellular relay networks with minimum rate constraints. 2014 IEEE International Conference on Acoustics, Speech and Signal Processing (ICASSP), May 2014, Florence, Italy. pp.7014 7018, 10.1109/ICASSP.2014.6854960 . hal-01098829v2

\section{HAL Id: hal-01098829 \\ https://hal.science/hal-01098829v2}

Submitted on 17 Jan 2015

HAL is a multi-disciplinary open access archive for the deposit and dissemination of scientific research documents, whether they are published or not. The documents may come from teaching and research institutions in France or abroad, or from public or private research centers.
L'archive ouverte pluridisciplinaire HAL, est destinée au dépôt et à la diffusion de documents scientifiques de niveau recherche, publiés ou non, émanant des établissements d'enseignement et de recherche français ou étrangers, des laboratoires publics ou privés. 


\title{
DISTRIBUTED ENERGY-EFFICIENT POWER OPTIMIZATION IN CELLULAR RELAY NETWORKS WITH MINIMUM RATE CONSTRAINTS
}

\author{
Giacomo Bacci ${ }^{\star \dagger} \quad$ E. Veronica Belmega $\quad$ Luca Sanguinetti ${ }^{\star \ddagger}$ \\ ${ }^{\star}$ Dipartimento di Ingegneria dell'Informazione, University of Pisa, Pisa, Italy, and CNIT, Parma, Italy \\ ${ }^{\dagger}$ Princeton University, Dept. Electrical Engineering, Princeton, NJ, USA

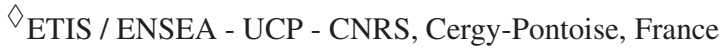 \\ ¥Alcatel-Lucent Chair, Ecole supérieure d'électricité (Supélec), Gif-sur-Yvette, France
}

\begin{abstract}
In this work, we derive a distributed power control algorithm for energy-efficient uplink transmissions in interferencelimited cellular networks, equipped with either multiple or shared relays. The proposed solution is derived by modeling the mobile terminals as utility-driven rational agents that engage in a noncooperative game, under minimum-rate constraints. The theoretical analysis of the game equilibrium is used to compare the performance of the two different cellular architectures. Extensive simulations show that the shared relay concept outperforms the distributed one in terms of energy efficiency in most network configurations.
\end{abstract}

\section{INTRODUCTION}

The use of fixed relays to convey messages from the base station (BS) to user equipments (UEs) through multihop communications is nowadays considered by many upcoming wireless standards (such as the 3GPP's LTE-Advanced [1]) as one of the most promising solutions to enhance cell-edge performance in cellular networks. One of the key challenges is to properly mitigate the intercell interference that arises among relays belonging to different neighboring cells. A possible solution is represented by the shared relay concept, originally proposed in IEEE $802.16 \mathrm{~m}$ [2], that relies on the idea of placing a multiple-antenna relay at the intersection of two or more cells. The relay decodes the signals from the multiple users in neighboring cells using the multiple receive antennas to cancel interference, and then retransmits to the BSs of different cells using broadcast methods. Refs. [3-6] show that shared relays achieve a substantial improvement in terms of spectral efficiency compared to multiple relays placed within each macrocell. To the best of our knowledge, all works available in the literature focus on measuring the spectral effi-

The research leading to these results has received funding from the People Programme (Marie Curie Actions) of the European Unions FP7 under REA Grant agreements no. PIOF-GA-2011-302520 GRAND-CRU and PIEF-GA-2012-330731 Dense4Green, and from the European Commission in the framework of the FP7 Network of Excellence in Wireless COMmunications NEWCOM\# (Grant agreement no. 318306). ciency of the two different architectures, whereas the problem of energy-efficient communications is not taken into account. However, the latter is becoming increasingly important as battery technology has not kept up with the increasing requirements stemming from ubiquitous multimedia applications. This is witnessed by the large number of works accounting for the cost of energy in the performance metrics. To this aim, the concept of link capacity per unit cost, originally proposed in [7], has been widely adopted in many different contexts (see for example [8-11] and references therein).

This work aims at revisiting the above concept to evaluate the energy efficiency of an uplink power control algorithm operating in conjunction with either multiple or shared relays. Unlike [3-6], we allow the multiple relays to interact in a distributed manner, by modeling the UEs as utility-driven rational agents that engage in a noncooperative game [12], using their own local information while satisfying qualityof-service (QoS) requirements in terms of minimum achievable rates. Note that a similar framework has been recently adopted in $[11,13]$ to study a $K$-user $N$-parallel Gaussian interference channel. Elaborating on this formulation, here we introduce the QoS requirements in the game, that thus becomes a generalized one $[12,14]$, also providing a simple method to compute the equilibrium point as a function of the network parameters using a fixed-point system of equations.

\section{SYSTEM AND SIGNAL MODEL}

\subsection{System model}

We consider the uplink of an arbitrary hexagonal orthogonal frequency-division multiple-access (OFDMA) multicellular network with universal frequency reuse. Each cell is divided into an inner and outer sector. The macro BS is placed at the cell center, whereas the outer sector is further divided into a certain number of adjacent sectors that are served using two different architectures. The first one, depicted in Fig. 1(a), refers to a network in which each sector is served by a fixed relay. The second one, shown in Fig. 1(b), accounts for a network in which the three adjacent sectors are served 


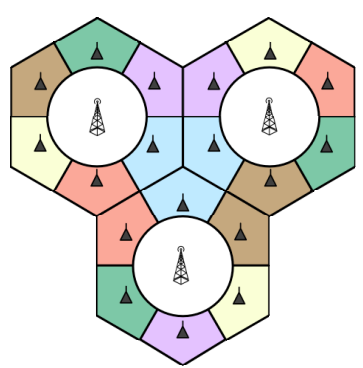

(a) multiple relays

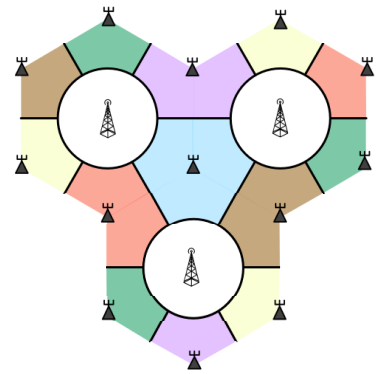

(b) shared relay
Fig. 1. Cellular relay network configurations.

by a shared relay placed at their intersection. In both cases, the UEs are assumed to be uniformly distributed within the cell, and equipped with single antennas, and perfect channel knowledge is assumed at the relays. The communication between the BS and the UEs takes place in a single-hop if the UEs are placed within the inner sector, whereas a two-hop protocol is needed if the UEs are within the outer sector. In the latter case, the information flows from the UE to the closest relay, and then from the relay to the BS. UEs within the same cell use orthogonal subcarriers to avoid mutual interference. However, interference may arise among UEs in adjacent clusters of neighboring cells sharing the same subcarrier.

To make the problem tractable, here we focus on one subcarrier only, that is still expedient to draw some insights on the problem at hand. Extensions to the multiple-carrier case are discussed in Section 5. Without loss of generality, let us focus on three adjacent clusters sharing the same subcarrier, and let $K$ and $M$ denote the total number of users simultaneously active over the same subcarrier within the three adjacent sectors, and the number of users served by each relay, respectively. Clearly, $K \in\{1,2,3\}$, and $M=1$ in the case of distributed relays, and $M=K$ when the (only) relay is shared across the cell sectors. For a fair comparison, the same number of antennas is employed in the two architectures. This means that, if we denote by $D$ the number of receiving antennas for each relay in the scenario of Fig. 1(a), the shared relay of Fig. 1(b) will be equipped with $M D$ receiving antennas.

\subsection{Signal model}

Let $\mathbf{h}_{k i} \in \mathbb{C}^{M D \times 1}$ denote the uplink channel vector whose entries $\left[\mathbf{h}_{k i}\right]_{m}$ represent the channel gains from the $i$ th UE to the $m$ th receive antenna of user $k$ 's serving relay. Denoting by $\mathbf{x}_{k}$ the vector collecting the samples received at UE $k$ 's serving relay, we may write $\mathbf{x}_{k}=\sum_{i=1}^{K} \mathbf{h}_{k i} \sqrt{p_{i}} s_{i}+\mathbf{w}_{k}$, where $p_{i}$ and $s_{i}$ denote UE $i$ 's transmit power and data symbol, respectively, whereas $\mathbf{w}_{k} \in \mathbb{C}^{M D \times 1}$ is a Gaussian vector accounting for the additive noise and possibly other sources of interference, with zero mean and covariance matrix $\sigma^{2} \mathbf{I}_{M D}$, where $\mathbf{I}_{L}$ is the $L \times L$ identity matrix. To keep the complexity of the relay at a tolerable level, a simple linear detec- tion scheme is employed for data detection. This means that the entries of $\mathbf{x}_{k}$ are linearly combined to form $y_{k}=\mathbf{g}_{k}^{H} \mathbf{x}_{k}$, where $\mathbf{g}_{k}$ is the vector employed for recovering the data of a generic user $k$. The signal-to-interference-plus-noise ratio (SINR) achieved by user $k$ at its serving relay takes the form

$$
\gamma_{k}(\mathbf{p})=\frac{\left|\mathbf{g}_{k}^{H} \mathbf{h}_{k k}\right|^{2} p_{k}}{\left\|\mathbf{g}_{k}\right\|^{2} \sigma^{2}+\sum_{i \neq k}\left|\mathbf{g}_{k}^{H} \mathbf{h}_{k i}\right|^{2} p_{i}}=\mu_{k}\left(\mathbf{p}_{\backslash k}\right) p_{k}
$$

where we explicitly report the dependence on the vector $\mathbf{p}=$ $\left[p_{1}, \ldots, p_{K}\right]^{T}=\left[p_{k}, \mathbf{p}_{\backslash k}\right]$, with $\mathbf{p}_{\backslash k}=\mathbf{p} \backslash p_{k}$ denoting the vector of all powers except user $k$ 's one. Using (1), user $k$ 's achievable data rate equals $r_{k}(\mathbf{p})=B \log _{2}\left(1+\gamma_{k}(\mathbf{p})\right)$, where $B$ is the subcarrier bandwidth.

When multiple relays are used, a maximum ratio combing (MRC) technique is employed. This amounts to setting $\mathbf{g}_{k}=\mathbf{h}_{k k}$, which requires no channel knowledge of the interfering users within neighboring cells. On the other hand, when a shared relay is employed, we resort to a zero-forcing (ZF) strategy, by setting $\mathbf{g}_{k}$ equal to the $k$ th column of the matrix $\mathbf{H}_{k}\left(\mathbf{H}_{k}^{H} \mathbf{H}_{k}\right)^{-1}$ with $\mathbf{H}_{k}=\left[\mathbf{h}_{k, 1}, \mathbf{h}_{k 2}, \ldots, \mathbf{h}_{k K}\right] \in$ $\mathbb{C}^{M D \times K}$. As seen, the computation of $\mathbf{H}_{k}$ requires knowledge of all $\mathbf{h}_{k i}$ for $i \neq k$. This can easily be acquired by the shared relay using conventional estimation schemes [3].

\section{ENERGY-EFFICIENT POWER OPTIMIZATION}

An accurate modeling of the system energy consumption is of primary importance when dealing with energy efficiency. To this end, note that, beside the radiative powers $\mathbf{p}$ at the output of the RF circuit, UEs also incur circuit power consumption during transmission. The overall power consumption $p_{T, k}$ of the $k$ th UE is thus given by $p_{T, k}=p_{k}+p_{c}$, where $p_{c}$ represents the average current power consumed by the device electronics, which is assumed to be independent of the transmission state and equal for all UEs. Following [13], the energy efficiency of the link can be measured by the utility function

$$
u_{k}(\mathbf{p})=\frac{r_{k}(\mathbf{p})}{p_{T, k}}=\frac{B \log _{2}\left(1+\gamma_{k}(\mathbf{p})\right)}{p_{k}+p_{c}} .
$$

Observe that, in cellular networks, UEs are usually required to satisfy $r_{k}(\mathbf{p}) \geq B \varphi_{k}$, where $\varphi_{k} \geq 0$ are the minimum normalized data rates (measured in $\mathrm{b} / \mathrm{s} / \mathrm{Hz}$ ).

To sum up, the design of an energy-efficient power allocation scheme requires to solve the optimization problem ${ }^{1}$ :

$$
\begin{aligned}
\mathbf{p}^{\star}=\underset{\mathbf{p}}{\arg \max _{\mathbf{p}}} & \sum_{k=1}^{K} u_{k}(\mathbf{p}) \\
\text { subject to } & p_{k} \geq 0 \quad \text { and } \quad r_{k}(\mathbf{p}) / B \geq \varphi_{k}
\end{aligned}
$$

whose solution is hard to compute, given the non-convex form of (3) caused by the QoS constraints.

\footnotetext{
${ }^{1}$ For analytical convenience, no upper bound on $p_{k}$ is considered, as (2) inherently prevents the UEs from using unnecessarily high power levels.
} 
To tackle with this, we investigate a distributed scenario in which every UE is a rational entity that chooses its transmit power to optimize its own link energy efficiency, measured by $u_{k}(\mathbf{p})$ in (2). The natural framework to model this kind of interactions is offered by non-cooperative game theory [12]. In particular, the underlying game $\mathcal{G}$ is defined as the tuple $\mathcal{G}=\left[\mathcal{K},\left\{\mathcal{A}_{k}\right\},\left\{u_{k}\right\}\right]$, in which: $\mathcal{K}=\{1,2, \ldots, K\}$ is the player set; $\mathcal{A}_{k}$ denotes the strategy set for which the constraints in (3) are satisfied; and $u_{k}$ is player $k$ 's payoff function defined in (2). Note that user $k$ 's action set depends on the actions of the other players, i.e., $\mathcal{A}_{k}=\mathcal{A}_{k}\left(\mathbf{p}_{\backslash k}\right)$, because of the rate constraint $r_{k}\left(\left[p_{k}, \mathbf{p}_{\backslash k}\right]\right) / B \geq \varphi_{k}$. In this case, the solution concept is the generalized Nash equilibrium (GNE) [14], that collects all system states that are stable to unilateral deviations [12].

\subsection{Feasibility of the GNE problem}

The feasibility of the GNE problem amounts to providing conditions ensuring that the overall action set $\mathcal{A}=\times_{k \in \mathcal{K}} \mathcal{A}_{k}$ is not empty. For later convenience, let us define a matrix $\mathbf{F} \in \mathbb{C}^{K \times K}$ whose $(k, i)$-th element is

$$
[\mathbf{F}]_{k, i}= \begin{cases}0 & i=k \\ \frac{\gamma_{k}^{\mathrm{req}}\left|\mathbf{g}_{k}^{H} \mathbf{h}_{k i}\right|^{2}}{\left|\mathbf{g}_{k}^{H} \mathbf{h}_{k k}\right|^{2}} & i \neq k\end{cases}
$$

with $\gamma_{k}^{\text {req }}=2^{\varphi_{k}}-1$ being the SINR such that $r_{k}=B \varphi_{k}$. Let $\rho_{\mathbf{A}}$ also denote the spectral radius of a generic matrix $\mathbf{A}$ [15].

Lemma 1 The problem (3) is feasible if and only if $\rho_{\mathbf{F}}<1$.

Proof: The proof is articulated in two steps. First, we provide a necessary and sufficient condition under which the minimal target rates are met with equality for all users. This amounts to proving the existence of a vector $\mathbf{p}^{\text {req }}=\left[p_{1}^{\text {req }}, \ldots, p_{K}^{\text {req }}\right]^{T}$, with $p_{k}^{\text {req }} \geq 0$, such that

$$
\frac{\left|\mathbf{g}_{k}^{H} \mathbf{h}_{k k}\right|^{2} p_{k}^{\text {req }}}{\left\|\mathbf{g}_{k}\right\|^{2} \sigma^{2}+\sum_{i=1, i \neq k}^{K}\left|\mathbf{g}_{k}^{H} \mathbf{h}_{k i}\right|^{2} p_{i}^{\text {req }}}=\gamma_{k}^{\text {req }}
$$

for any $k \in \mathcal{K}$. By rearranging the terms, we rewrite (5) as

$$
\left(\mathbf{I}_{K}-\mathbf{F}\right) \mathbf{p}^{\text {req }}=\mathbf{s}
$$

where $\mathbf{s} \in \mathbb{C}^{K \times 1}$ is a vector with positive elements $[\mathbf{s}]_{k}=$ $\gamma_{k}^{\text {req }}\left\|\mathbf{g}_{k}\right\|^{2} \sigma^{2} /\left|\mathbf{g}_{k}^{H} \mathbf{h}_{k k}\right|^{2}$. Since $\mathbf{F}$ is irreducible and nonnegative, from [15, Theorem 2.1] it follows that a solution $\mathbf{p}^{\text {req }} \geq \mathbf{0}$ to (6) exists for any $\mathbf{s} \geq \mathbf{0}$ if and only if $\rho_{\mathbf{F}}<1$.

For the second step, we have to prove that $\rho_{\mathbf{F}}<1$ is also necessary and sufficient for the existence of a vector $\tilde{\mathbf{p}}=$ $\left[\tilde{p}_{1}, \ldots, \tilde{p}_{K}\right]^{T}$, with $\tilde{p}_{k} \geq 0$, such that $\gamma_{k}(\tilde{\mathbf{p}})=\tilde{\gamma}_{k} \geq \gamma_{k}^{\text {req }}$ for all $k \in \mathcal{K}$. While the sufficiency follows trivially, proving the necessity is more elaborated. Assume that such $\tilde{\mathbf{p}} \geq \mathbf{0}$ exists. Then, the spectral radius of the matrix $\tilde{\mathbf{F}}$, defined as in (4) by replacing $\gamma_{k}^{\text {req }}$ with $\tilde{\gamma}_{k}$, is such that $\rho_{\tilde{\mathbf{F}}}<1$. Since $\mathbf{F} \leq \tilde{\mathbf{F}}$, then $\rho_{\mathbf{F}} \leq \rho_{\tilde{\mathbf{F}}}[16]$ and, thus, $\rho_{\mathbf{F}}<1$. In conclusion, a vector $\mathbf{p}^{\text {req }} \geq \mathbf{0}$ satisfying (6) always exists, and hence the necessity.

\subsection{Analysis of the GNE}

In this subsection, we focus on the analysis of the outcome of the game $\mathcal{G}$. Let us denote by $W(\cdot)$ the Lambert function [17] defined such that $z=W(z) \cdot \exp (W(z))$ for any $z \in \mathbb{C}$. As stated in the following theorem, it turns out that, whenever Lemma 1 holds, $\mathcal{G}$ has a unique solution.

Theorem 1 If the GNE problem is feasible, then $\mathcal{G}$ has a unique GNE, achieved by the unique solution $\mathbf{p}^{\star} \triangleq$ $\left[p_{1}^{\star}, \ldots, p_{K}^{\star}\right]$ to the fixed-point system of equations

$$
p_{k}^{\star}=\frac{\gamma_{k}^{\star}\left(\mathbf{p}_{\backslash k}^{\star}\right)}{\mu_{k}\left(\mathbf{p}_{\backslash k}^{\star}\right)}
$$

where $\mu_{k}\left(\mathbf{p}_{\backslash k}^{\star}\right)$ is defined as in (1) and the optimal SINR $\gamma_{k}^{\star}\left(\mathbf{p}_{\backslash k}^{\star}\right)$ is given by $\gamma_{k}^{\star}\left(\mathbf{p}_{\backslash k}^{\star}\right)=\max \left\{\gamma_{k}^{\text {req }}, \tilde{\gamma}_{k}\left(\mathbf{p}_{\backslash k}^{\star}\right)\right\}$ with

$$
\tilde{\gamma}_{k}\left(\mathbf{p}_{\backslash k}^{\star}\right)=\exp \left[W\left(\frac{\mu_{k}\left(\mathbf{p}_{\backslash k}^{\star}\right) p_{c}-1}{e}\right)+1\right]-1 .
$$

Due to space limitations, we can only give the main guidelines of the proof (see [18] for more details). The existence follows from the topology of the overall action set $\mathcal{A}_{k}$ [14], interpreted as a point-to-set mapping [19], jointly with the continuity and quasi-concavity properties of the payoff function $u_{k}(\cdot)$ as a function of $p_{k}$ for all $k \in \mathcal{K}$ [12]. To prove the uniqueness, we use the best-response correspondence [12], described by (7), which ensures that its fixed point is the unique GNE of the underlying game. Since (7) turns out to be a standard function [20] (i.e., component-wise positive, monotonous, and scalable), it has a unique fixed-point [21]. This amounts to saying that the GNE is unique. Note that, unlike other game-theoretic power control schemes (e.g., [22-24]), here not only the optimal power $p_{k}^{\star}$, but also the optimal SINR $\gamma_{k}^{\star}$ depends on the others' actions $\mathbf{p}_{\backslash_{k}}^{\star}$ through $\tilde{\gamma}_{k}$. This feature makes proving that (7) is a standard function rather challenging.

\section{PERFORMANCE ASSESSMENT}

Using the theoretical results derived in Section 3, we are interested in comparing the performance of the two relay configurations illustrated in Section 2.1, by means of an extensive simulation campaign. Throughout the simulations, we assume $K=3$. To include the impact of spatial correlation among the $M D$ colocated antennas, the channel power gains are computed as $\mathbf{h}_{k i}=\Theta^{1 / 2} \boldsymbol{\zeta}_{k i}$, where $\Theta$ is a square matrix of size $D M$ and elements $[\Theta]_{k, i}=\theta^{|k-i|}$, with $\theta$ denoting the correlation index between adjacent antennas [25]. To simulate the effects of fading and shadowing, the coefficients $\left[\boldsymbol{\zeta}_{k j}\right]_{m}$ are modeled as zero-mean statistically independent and circularly symmetric Gaussian random variables with variance $\left(R / d_{k i}\right)^{\varsigma}$, where $R$ is the cell radius, $d_{k i}$ is the distance between the $k$ th UE and the UE $i$ 's receiving relay, and $\varsigma=4$ is the path-loss exponent. 


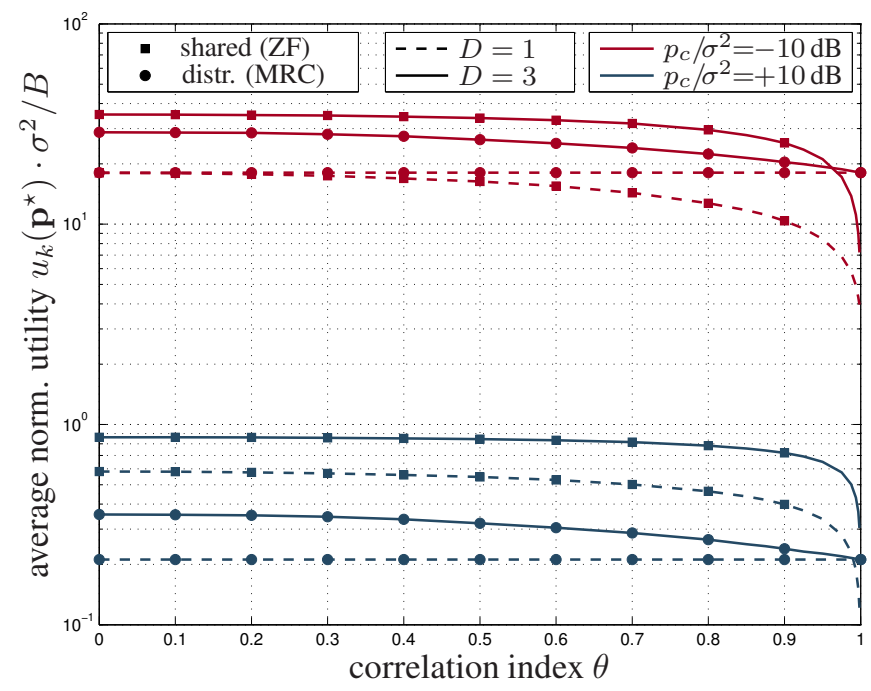

Fig. 2. Average norm. utility at the GNE as a function of $\theta$.

To average over all possible positions of the UEs within each sector, we use 10, 000 independent realizations of a feasible scenario, where the reference user is placed randomly in the observed vector at a normalized distance $\xi \in[d / R, 1]$, where $d=R / 2$ is the radius of the single-hop region, whereas the other two interfering users are placed randomly within their reference sectors. To account for all positions $\xi$, we use a discretized version of the range $[d / R, 1]$, using a quantization step $\Delta \xi=0.02$, and we average using the theoretical probability density function $f_{\Xi}(\xi)$, derived in [18] for a uniform distribution of UEs across the two-dimensional plane covered by the multicellular system under investigation. The shared relay is placed at the intersection of the three cells, as represented in Fig. 1(b), whereas the distributed relays are placed so as to minimize the average UE transmit power [18]. To include the impact of variable QoS constraints, the reference user adopts $\varphi_{k}=1.5 \mathrm{~b} / \mathrm{s} / \mathrm{Hz}$, whereas the other two UEs select a random $\varphi_{i}$ uniformly distributed in $[0,3] \mathrm{b} / \mathrm{s} / \mathrm{Hz}$.

In Figs. 2-3, we evaluate the performance in terms of energy efficiency of the two architectures as a function of the antenna correlation $\theta$. Dashed and solid lines depict the case when $D=1$ and $D=3$, respectively, whereas square and circular markers report the results obtained with the shared relay using $\mathrm{ZF}$, and the multiple relays using MRC, respectively. Both transmit powers and circuit powers are normalized with respect to the noise power $\sigma^{2}$. In particular, red lines correspond to the case $p_{c} / \sigma^{2}=-10 \mathrm{~dB}$, in which the circuit power is negligible with respect to the noise, whereas blue lines report the case $p_{c} / \sigma^{2}=+10 \mathrm{~dB}$, in which the circuit power is significantly higher than the noise.

Fig. 2 reports the average normalized utility for the cases introduced above. As can be seen, the MRC-based distributed-relay configuration outperforms the ZF-based shared-relay solution only when the system is noise-limited. This is due to the fact that, when $p_{c} \ll \sigma^{2}$, also $p_{k}^{\star} \ll \sigma^{2}$,

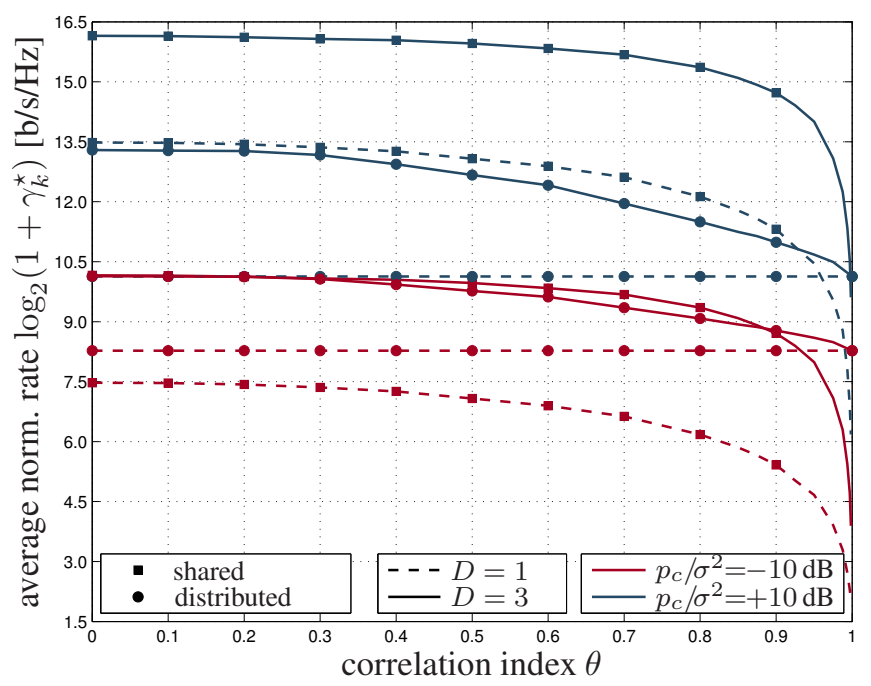

Fig. 3. Average norm. rate at the GNE as a function of $\theta$.

due to the increasing behavior of $p_{k}^{\star}$ with respect to $p_{c}$ [18], and thus the system is noise-limited. In addition to this, when the number of antennas is limited (notably, $D=1$ and thus $M D=3$ ), the improvement brought by the multiple antennas at the shared relay is not sufficiently effective. In all other cases, the shared relay is shown to outperform the distributed relay configuration for reasonable values of $\theta$.

For the sake of completeness, Fig. 3 reports the average normalized rate at the GNE as a function of $\theta$, yielding similar conclusions. It is worth mentioning that the minimum rate $\varphi_{k}=1.5 \mathrm{~b} / \mathrm{s} / \mathrm{Hz}$ is met not only on average, but also at every realization of the simulation.

\section{CONCLUSIONS}

We have studied the performance of a relay-based cellular network using an energy-efficient uplink power control, formulated in terms of achieved rate per unit of energy consumed, also considering the impact of the circuit power. To this aim, we have considered two different relay architectures: a multiple-antenna shared relay that serves multiple macrocells, and a set of distributed relays, each serving a sector within one macrocell. Numerical results have revealed that the distributed-relay solution outperforms the shared relay only when the system is noise-limited. In all other cases, the benefits given by multiuser cancellation increase as the impact of multiple access interference increases. Interestingly, this conclusion is in line with other recent studies (e.g., [3-6]) focusing instead on spectral efficiency. Moreover, although here we focused on a relay-based network, the analysis can be applied to many other scenarios, such as small cells [26]. Future work is needed to assess the performance when considering a multicarrier system, thus also introducing subcarrier allocation, and also measuring the receiver computational costs, and the installation and maintenance costs. 


\section{REFERENCES}

[1] A. Ghosh, R. Ratasuk, B. Mondal, N. Mangalvedhe, and T. Thomas, "LTE-advanced: Next-generation wireless broadband technology," IEEE Wireless Commun., vol. 17, no. 3, pp. 10-22, Jun. 2010.

[2] IEEE 802.16 Broadband Wireless Access Working Group, "IEEE Standard for Local and metropolitan area networks - Part 16: Air Interface for Broadband Wireless Access Systems - Amendment 3: Advanced Air Interface," Tech. Rep. IEEE 802.16m-2011, 2011.

[3] S. W. Peters, A. Y. Panah, K. T. Truong, and R. W. Heath, "Relay architectures for 3GPP LTEadvanced," EURASIP J. Wireless Commun. Networking, vol. 2009:618787, 2009.

[4] A. Y. Panah, K. T. Truong, S. W. Peters, and R. W. Heath, "Interference management schemes for the shared relay concept," EURASIP J. Wireless Commun. Networking, vol. 2011:269817, 2011.

[5] Y. Lin and W. Yu, "Fair scheduling and resource allocation for wireless cellular network with shared relays," IEEE J. Select. Areas Commun., vol. 30, no. 8, pp. 1530-1540, Sep. 2012.

[6] A. Adhikary, J. Nam, and G. Caire, "Joint spatial division and multiplexing - The large-scale array regime," IEEE Trans. Information Theory, vol. 59, no. 10, pp. 6441-6463, Oct. 2013.

[7] S. Verdú, "On channel capacity per unit cost," IEEE Trans. Information Theory, vol. 36, no. 5, pp. 10191030, Sep. 1990.

[8] E.-V. Belmega and S. Lasaulce, "Energy-efficient precoding for multiple-antenna terminals," IEEE Trans. Signal Process., vol. 59, no. 1, pp. 329-340, Jan. 2011.

[9] C. Isheden, Z. Chong, E. Jorswieck, and G. Fettweis, "Framework for link-level energy efficiency optimization with informed transmitter," IEEE Trans. Wireless Commun., vol. 11, no. 8, pp. 2946-2957, Aug. 2012.

[10] R. Xie, F. Yu, H. Ji, and Y. Li, "Energy-efficient resource allocation for heterogeneous cognitive radio networks with femtocells," IEEE Trans. Wireless Commun., vol. 11, no. 11, pp. 3910-3920, Nov. 2012.

[11] G. Miao, N. Himayat, G. Li, and S. Talwar, "Distributed interference-aware energy-efficient power optimization," IEEE Trans. Wireless Commun., vol. 10, no. 4, pp. 1323-1333, Apr. 2011.

[12] D. Fudenberg and J. Tirole, Game Theory. Cambridge, MA: MIT Press, 1991.
[13] G. Miao, N. Himayat, and G. Li, "Energy-efficient link adaptation in frequency-selective channels," IEEE Trans. Commun., vol. 58, no. 2, pp. 545-554, Feb. 2010.

[14] F. Facchinei and C. Kanzow, "Generalized Nash equilibrium problems," Quarterly J. Operations Research, vol. 5, no. 3, pp. 173-210, Sep. 2007.

[15] E. Seneta, Non-negative Matrices and Markov Chains, 3rd ed. New York, NY: Springer, 2006.

[16] R. A. Horn and C. R. Johnson, Matrix Analysis, 2nd ed. Cambridge, UK: Cambridge University Press, 1990.

[17] R. Corless, G. Gonnet, D. Hare, D. Jeffrey, and D. Knuth, "On the Lambert W function," Advances in Computational Mathematics, vol. 5, pp. 329-359, 1996.

[18] G. Bacci, E.-V. Belmega, and L. Sanguinetti, "Energyefficient power control for relay-based multicellular networks," Tech. Rep., Oct. 2013. [Online]. Available: http://www.iet.unipi.it/l.sanguinetti/relay.pdf

[19] W. Hogan, "Point-to-set maps in mathematical programming," SIAM Review, vol. 15, no. 3, pp. 591-603, Jul. 1973.

[20] R. D. Yates, "A framework for uplink power control in cellular radio systems," IEEE J. Select. Areas Commun., vol. 13, no. 9, pp. 1341-1347, Sep. 1995.

[21] M. Schubert and H. Boche, "Solution of the multi-user downlink beamforming problem with individual SINR constraints," IEEE Trans. Veh. Technol., vol. 53, no. 1, pp. 18-28, Jan. 2004.

[22] C. U. Saraydar, N. B. Mandayam, and D. J. Goodman, "Pricing and power control in a multicell wireless data network," IEEE J. Sel. Areas Commun., vol. 19, no. 10, pp. 1883-1892, Oct. 2001.

[23] G. Bacci and M. Luise, "A game-theoretic perspective on code synchronization for CDMA wireless systems," IEEE J. Sel. Areas Commun., vol. 30, no. 1, pp. 107118, Jan. 2012.

[24] G. Bacci, L. Sanguinetti, M. Luise, and H. V. Poor, "A game-theoretic approach for energy-efficient contention-based synchronization in OFDMA systems," IEEE Trans. Signal Process., vol. 61, no. 5, pp. 12581271, Mar. 2013.

[25] D. Tse and P. Viswanath, Fundamentals of Wireless Communications. Cambridge, UK: Cambridge University Press, 2005.

[26] G. Bacci, E. V. Belmega, and L. Sanguinetti, "Distributed energy-efficient power and subcarrier allocation for OFDMA-based small cells," in Proc. IEEE Intl. Conf. Commun. (ICC), Sydney, Australia, Jun. 2014. 